\title{
EL MARCO DE REFERENCIA PARA LA FUSION ENTRE RADIO NACIONAL DE ESPAÑA Y RADIO CADENA ESPAÑOLA
}

\author{
THE FRAMEWORK FOR THE FUSION BETWEEN RADIO \\ NACIONAL DE ESPAÑA AND RADIO CADENA ESPAÑOLA
}

\begin{abstract}
AUTOR
Miguel Ángel Ortiz Sobrino

Profesor de Periodismo en la Facultad de Ciencias de la Información de la Universidad Complutense de Madrid (España)

maortiz.@ccinf.ucm.es
\end{abstract}

\section{RESUMEN}

En julio de 1988 el Consejo de Ministros español aprobó la fusión entre las dos cadenas de radio pertenecientes al Ente Publico RTV. En aquella época, la radio, la televisión y la publicidad experimentaron importantes transformaciones .Durante el período 1988-1990, la radio asistió a la promulgación de una serie de leyes y normas que irían dibujando el nuevo modelo de radiodifusión español. En el sector de la televisión, el modelo público ya había entrado en crisis. La petición de una televisión propia por parte de las distintas nacionalidades se dio por zanjada con la creación, en 1989, de cuatro nuevos canales de televisión autonómico. Por su parte, la publicidad encontró con la ampliación del mapa televisivo autonómico $y$, en consecuencia, con una audiencia fragmentada, que se segmentaba por preferencias de programación, y no en estratos diferenciados por la capacidad de compra de los espectadores. 
Conviene resaltar que, desde 1982, la audiencia de la radio española había experimentado un continuo descenso. Las estimaciones sobre la pérdida de oyentes desde 1982 hasta 1988 se cifraba en casi nueve puntos porcentuales, lo que equivalía a que uno de cada siete oyentes había dejado de serlo. De acuerdo con el análisis de Eduardo García Matilla sobre la audiencia de radio durante 1988, las pérdidas de oyentes de la Onda Media en el período 1982-1988 podía calificarse de espectacular, puesto que la caída alcanzaba un $20 \%$ y situaba la audiencia, en 1988 , en el 21,45 de la población.

\section{PALABRAS CLAVE}

Radio - Televisión - Publicidad - Audiencia - Fusión mercantil.

\section{ABSTRACT}

In July 1988 the Spanish Council of Ministers approved the merger between the two radio stations belonging to the Ente Publico RTV. At that time, radio, television and advertising experienced significant transformations. During the period 1988-1990, the radio went to the enactment of a series of laws and regulations that would draw the new model of Spanish broadcasting. In the television sector, the public model was already in crisis. The request for a television of their own by different nationalities gave settled with the creation in 1989 of four new regional television channels. For his part, found advertising to the expansion of regional TV map, and therefore, with a fragmented audience, which is segmented by programming preferences, not differentiated strata purchasing power of the spectators. It should be noted that since 1982, the Spanish radio audience had experienced a steady decline. Estimates of the loss of listeners from 1982 to 1988 was estimated at nearly nine percentage points, equivalent to one in seven had ceased to be listeners. According to Eduardo Garcia Matilla analysis of the radio audience in 1988, losses of 
medium wave listeners in the period $1982-1988$ could be described as spectacular as the fall reached $20 \%$ and placed the audience in $1988,21.45$ of the population.

\section{KEY WORDS}

Radio - Television - Advertising - Audience - Commercial Fusion

\section{ÍNDICE}

1.- La fusión de Radiocadena Española y Radio Nacional de España

2. El entorno mediático de la fusión: 1988-1990: un marco de referencia

2.1. La radio y su entorno

2.2. El marco legal de la radiodifusión

2.3. La concentración de emisoras

2.4. Los grupos multimedia y la radio

2.5. La audiencia de la radio

2.6. La programación radiofónica

3. El entorno social e informativo para la fusión RCE/RNE

\subsection{Aspectos sociales}

3.2. El mundo laboral

3.3. Niveles de instrucción y marginación

3.4. Aspectos informativos

3.4.1. Internacional 


\subsubsection{Nacional}

4. Bibliografía

\section{1.- La fusión de Radiocadena Española y Radio Nacional de España}

En el verano de 1988, los principales diarios españoles daban cuenta de la publicación en el BOE del Real Decreto $895 / 188$, de 20 de julio, por el que se regulaba la fusión de Radio nacional de España Y Radiocadena Española.

El Consejo de Ministros, en reunión celebrada el 20 de julio de 1988, aprobaba la fusión de estas dos emisoras de radio dependientes del Ente Público RTVE. De este modo, la nueva Radio Nacional de España resultado de la fusión se convertía en el primer grupo radiofónico español, en cuanto a número de emisoras, recursos humanos y presupuesto.

La nueva emisora, contaría a partir de entonces con cinco ofertas diferenciadas:

Radio 1, Radio Clásica, Radio 3, Radio 4 y Radio 5. Además de las emisiones internacionales de radio Exterior. 


\section{El entorno mediático de la fusión: 1988-1990: un marco de referencia}

Pero, ¿en qué condiciones se produce la fusión de Radio Nacional y Radio Cadena Española? ¿Qué entorno se divisa desde el sector público de radiodifusión?

\subsection{La radio y su entorno}

En aquella época, la radio, la televisión y la publicidad experimentaron importantes transformaciones.

Durante el período 1988-1990, la radio asistió a la promulgación de una serie de leyes y normas que irían dibujando el nuevo modelo de radiodifusión español.

Así, la Ley de Ordenación de Telecomunicaciones (LOT), de diciembre de 1987, había establecido el marco global de la radio y su gestión. Después, recogiendo el espíritu de la LOT, el Plan Técnico Nacional de Frecuencia Modulada de febrero de 1989, trajo consigo la adjudicación de nuevas emisoras de FM a las cadenas comerciales ya establecidas, y a la radio pública.

El modelo empresarial también se reajusto por aquella época. Las cadenas privadas tendían a la concentración mediante absorciones o adquisiciones de emisoras. Igualmente en la radio pública, como se está describiendo, se produciría la fusión de Radio Nacional de España y Radiocadena Española. Pero la reconversión empresarial fue más allá, hasta tal punto que las cadenas de radio se iban integrando en grupos multimedia, presentes, además, en el sector de la prensa escrita y en el de la televisión. 
Mientras, la audiencia de radio experimentaba un continuo descenso - según se desprende el Estudio General de Medios de la época- y se producía el transvase de oyentes de Onda Media a la FM. A pesar de ello, las cadenas ofrecían el mismo tipo de programas: no había diferenciación; los mismo contenidos, a las mismas horas.

En el sector de la televisión, el modelo público ya había entrado en crisis. La petición de una televisión propia por parte de las distintas nacionalidades se dio por zanjada con la creación, en 1989, de cuatro nuevos canales de televisión autonómico.

Un año después, el monopolio televisivo de TVE se quiebra con el crecimiento de los tres primeros canales de televisión privada. No obstante, la televisión privada había visto frustrada una de sus iniciativas: el Canal 10, que comenzó sus emisiones en enero de 1988, suspendiéndolas en agosto de ese mismo año. Otros proyectos, como el video comunitario o la televisión por satélite, contaron, también, con u un importante desarro9llo durante el periodo 1988-1990.

Por su parte, la publicidad encontró con la ampliación del mapa televisivo autonómico $y$, en consecuencia, con una audiencia fragmentada, que se segmentaba por preferencias de programación, y no en estratos diferenciados por la capacidad de compra de los espectadores.

En un primer momento, la aparición de nuevos canales de televisión, produjo un estado de euforia en los agentes del sector publicitario que llegaron a prever un aumento del porcentaje de inversión en el medio, frente a la radio y la prensa escrita. Sin embargo, la cuota publicitaria de la televisión, descendió cuatro puntos porcentuales entre 1989 y 1990: los mismos que subieron, conjuntamente, diarios, los suplementos semanales y las revistas. El desplazamiento de la inversión publicitaria de la televisión a la prensa parecía responder al exceso de ofertas de espacios en las televisiones y a un abaratamiento de tarifas reales en las nuevas cadenas, que se traducía en una menor cuota de mercado. 


\subsection{El marco legal de la radiodifusión}

Como se apuntaba anteriormente, el modelo de la radiodifusión española se organizaba de acuerdo con la Ley de Ordenación de las Telecomunicaciones (LOT), aprobada el 18 de diciembre de 1987. Según la LOT, el Estado y sus Entes Públicos se reservaban la explotación directa de los servicios de radio en Onda Media y Onda Larga. Los servicios de Onda Media podían explotarse en concurrencia, por gestión directa del Estado o por gestión indirecta, mediante concesiones administrativas. La LOT señalaba que los servicios en Frecuencia Modulada, en ondas métricas, también podían ser explotados en concurrencia, ya fuera directamente por las administraciones públicas y sus Entes Públicos o, de manera indirecta, mediante la concesiona administrativa a personas físicas y jurídicas.

Las críticas a este marco global de la radio dispuesto en la LOT no se hicieron esperar. Mariano Cebrián Herreros ${ }^{1}$ destaca los ataques de la Federación Española de Radios Libres, que denunció "el reparto de poder de poder entre los grandes grupos e intereses particulares, mientras se dejaba sin apoyo a los grupos sociales que ellos representaban" (Cebrián Herreros, 1992, Pág 216).

La Asociación de Empresas de Radiodifusión Independiente aseguró que "con dicha ley sólo se beneficiarían las grandes cadenas previamente establecidas", mientras que la Asociación Española de Radiodifusión Privada (AERP) centraba su descontento en "la primacía que se daba al sector público".

Además de la LOT, la radio española de los últimos años de la década de los ochenta se ajustó al Plan Técnico Nacional de la Frecuencia Modulada, aprobado mediante Real Decreto el 10 de febrero de 1989. En este plan se recogían cuatro modelos de radiodifusión: la dependiente del Estado, la gestionada por los Entes

\footnotetext{
${ }^{1}$ CEBRIAN HERREROS, Mariano: "Radio pública/radio privada". En VV.AA. Comunicación Social: 1992. Tendencias. Fundesco. Madrid (pág. 216).
} 
correspondientes a las Comunidades Autónomas, la radiodifusión municipal, y las emisiones de gestión directa y privada.

Tras el concurso Público para las emisiones de FM, derivado del Plan Técnico Nacional, el reparto vino a reforzar a las cadenas privadas ya establecidas, cuya cobertura se vio notablemente mejorada. La radio pública también amplio su presencia nacional, tanto en los canales de programación de Radio Nacional de España (RNE) como en las distintas cadenas autonómicas. Del mismo modo, la radio municipal obtuvo un impulso en áreas de comunicación local.

La aprobación del Plan Técnico Nacional de la Frecuencia Modulada también estuvo rodeado de una importante polémica dirigida desde la Asociación Española de Radiodifusión Privada (AERP). La campaña de presión de la AERP tenía un doble objetivo: de un lado, poner en guardia a la Administración frente al fenómeno social de las radios libres y piratas; de otro denunciar las pretensiones del Estado de tener mayor presencia en las ondas. En el análisis de FUNDESCO, referido a $1989^{2}$, se recogían dos informaciones de prensa que ilustran la polémica desencadenada por el plan técnico al que nos venimos refiriendo.

Según Eugenio Galdón, Presidente de la AERP, la aprobación del Plan Técnico de la Frecuencia Modulada, era la peor noticia que se le ha podido dar a la radiodifusión privada e independiente de esta país desde que se consagró la libertad informativa de la radio. Así lo recogía el diario El País, en su edición del 15 de febrero de 1989.

Asimismo, para la Federación Española de radios Libres, las concesiones favorecían la concentración de poder en las cadenas comerciales. Según Esteban Ibarra, presidente de la Federación, la radio privada había sido el chivo expiatorio, a fin de ocultar el problema de fondo: el reparto de poder en la FM. El plan técnico Nacional de la Frecuencia Modulada, según la Federación, discriminaba objetivamente a las emisoras libres al no contemplar previsiones técnicas necesarias que garantizaban la

\footnotetext{
2 DIAZ-NOSTY, Bernardo. "La radio en España. Entre la crisis de identidad y los grandes beneficios". En VV.AA. Comunicación Social: 1989. Tendecias. Fundesco Madrid. (pág. 56).
} 
existencia de proyectos socioculturales no lucrativos. La controversia era recogida y tratada por el diario El País, el día 20 de febrero de 1989.

Al año de iniciarse la fusión de Radio Nacional y Radiocadena Española, otra ley, la de Organización y Control de las Emisoras de radiodifusión Sonora municipal (10/1991, de 8 de abril), completaría el marco legal dispuesto donde se contextualiza la fusión. Con esta ley la Administración justificaría la instalación de emisoras en las zonas del territorio nacional carentes de cobertura radiofónica de carácter local.

La financiación de este tipo de emisoras, que además de con los presupuestos locales podía llevarse a cabo con todo tipo de publicidad, suscitó un nuevo foco de críticas. El Partido popular calificó la ley de anacrónica y de "atentado contra la libre competencia". La Asociación Española de Radio Comercial (AERC), alertó del grave riesgo que corría la radio privada al señalar que sería imposible competir con más de mil nuevas emisoras que tendrían doble financiación.

Otras voces, sin embargo, han aplaudido esta ley. Según Rosa Franquet ${ }^{3}$, las críticas de la AERC eran infundadas, y en su opinión no podía existir competencia entre las radio privadas y las municipales.

"... si se produjese un incremento espectacular de las radios municipales, éstas tendrían que competir con una programación en cadena que potencia las estrellas, los tratamientos espectaculares y que dispone de gran cantidad de medios técnicos... La mayoría de las estaciones municipales se encuentran ubicadas en poblaciones pequeñas y poco rentables para las estrategias empresariales, que buscan la máxima concentración de población, lo que destaca de entrada una lucha desaforada por conseguir parte del pastel publicitario". (Franquet, 1991, Pág. 78)

\footnotetext{
${ }^{3}$ FRANQUET, Rosa; "Consolidación de las radios municipales". En VV.AA. Comunicación Social: 1991. Tendencias. Fundesco. Madrid (pá. 78).
} 


\subsection{La concentración de emisoras}

Durante los últimos años de la década de los ochenta se produjo una tendencia a la concentración de emisoras que afectó tanto a la radio pública como a la privada.

En la radio estatal, en 1989, se procede a la fusión de Radiocadena Española (RCE) y radio nacional de España (RNE). El resultado fue una nueva RNE con cinco canales y seis programaciones distintas.

Para Eduardo García Matilla ${ }^{4}$,

"el objetivo fundamental de esa fusión de RCE y RNE fue aunar los recursos técnicos y humanos para así poder mejorar las ofertas a la audiencia y racionalizar los sistemas de trabajo en los casi 80 centros de producción repartidos por las 17 comunidades autónomas". (García Matilla 1989, Pág. 143)

Mariano Cebrián ${ }^{5}$ señalaría después como gran error de la fusión no haber conseguido ampliar la oferta especializada de programaciones de la radio pública. Su propuesta era convertir los canales de RCE en emisoras autonómicas y locales mediante acuerdos con las autoridades correspondientes. Del resultado de la fusión destacaba -según Cebrián- a sobredotación de la plantilla de RNE, que incrementaba los problemas para resolver la crisis que venía padeciendo RTVE y, lo más grave aún, la falta de clarificación de los canales.

En la radio privada también se inició una tendencia hacia la concentración a partir de finales de la década de los ochenta. De estos movimientos empresariales destacamos la creación de una nueva cadena de ámbito nacional: Cadena Ibérica. Sus emisiones

\footnotetext{
${ }^{4}$ GARCIA MATILLA, Eduardo: "Un año de transición para la radio española". En VV.AA. Comunicación social: 1989. Tendencias Fundesco. Madrid. (pág. 143).

${ }^{5}$ CEBRIAN HERREROS, Mariano: "Radio pública/radio privada". En VV.AA. Comunicación Social: 1992. Tendencias. Fundesco. Madrid (pág. 218)
} 
empiezan en septiembre de 1988, a través de 22 emisoras asociadas -Radio España. Radio Miramar, Radio Heraldo de Aragón y Onda vasca, entre otras-, con cinco horas de programación en cadena, además de los servicios informativos. Radio España de Madrid producía el $80 \%$ de los espacios, y Radio Miramar de Barcelona el $20 \%$ restante.

También Radio 80, la cadena surgida de las primeras concesiones en FM de la década de los 80, es absorbida por Antena 3 para ser adquirida por el Grupo PRISA pocos años después.

En los años siguientes, la tendencia seguiría con la adquisición por parte de la Cadena COPE del 50\% de la catalana Cadena 13; la ONCE adquiría la Cadena RATO, formando una cadena de ámbito nacional con el indicativo Onda Cero; el Radio Minuto y Radio Dial, etc.

\subsection{Los grupos multimedia y la radio}

A la concentración de emisoras hay que añadir la tendencia de las cadenas de radio a integrarse en grupos multimedia. El caso más sobresaliente es el del Grupo PRISA. En 1990 y antes de la vinculación de Antena 3 Radio a dicho grupo, el presidente de PRISA $^{6}$ afirmaba aue las empresas de comunicación que no fueran multimedia eran inconcebibles, pues los mercados nacionales eran cada vez más pequeños. Según el representante de PRISA, Europa nos había cogido a trasmano, porque España había permanecido mucho tiempo fuera del contexto europeo, los mercados de otros países eran muy poderosos, y porque una visión pacata había retrasado el nacimiento de las televisiones privadas en nuestro país.

${ }^{6}$ El País, 12 junio. 1990. Pág. Comunicación. 
Pero no todas las empresas que recalaron en la radio procedían del sector de los medios de comunicación. La aparición de una entidad como la Organización Nacional de ciegos (ONCE) en el mercado de los medios de comunicación daba cuenta del interés de las inversiones en el sector para el fortalecimiento de la propia Organización de nacional Ciegos. En cualquier caso la tendencia discurría por senderos multimedia.

Así, la ONCE, aparte de la citada adquisición del $51 \%$ de la Cadena RATO, cuenta ya en 1990 con el $25 \%$ de las acciones de Tele 5. Por esas fechas, Antena 3 Radio aparece unida a Antena 3 televisión y vinculada a varios periódicos.

Por su parte, la Cadena SER, dentro del Grupo PRISA y con las absorciones de Radio Minuto y Radio Corazón, además de Radio El País, tenía una participación en la televisión privada -un 25\%- a través de Canal Plus.

La Cadena de Ondas Populares, la COPE, tampoco se quedó atrás. Mediante sus sociedades Editorial Catalana y Radio Sabadell, compró el 50\% de la cadena Cadena 13 y, además, se proyectó hacia el mercado de la producción video-gráfica con su empresa Iniciativas Radiofónicas y de Televisión, S.A.

La nueva estrategia del sector y su renovación empresarial vino acompañada de una incipiente conversión en la infraestructura tecnológica de las cadenas. El comienzo de esta innovación técnica la iniciaría la Cadena SER, el 22 de septiembre de 1988, con la distribución de sus programas vía satélite con sonido digital. Emilio Prieto ${ }^{7}$ vislumbro un cambio de actitud en las empresas radiofónicas al apostar por la modernización de sus infraestructuras. La renovación se encontraba asociada, según Prado, a las nuevas estructuras empresariales. Al referirse al salto dado por la SER, incidía en la renovación paralela del sistema de relaciones con las emisoras asociadas,

\footnotetext{
7 PRADO, Emilio: "La radio española ante el desafío del futuro". En VV.AA. Comunicación social: 1989. Tendencias. Fundesco. Madrid. (pág. 133).
} 
"que tenían unos estatutos tan variopintos como la peculiaridad de cada propietario y su relación histórica con las antiguas familias propietarias de la cadena, que imprimieron un sello paternalista a la gestión de la empresa, existiendo, incluso, acuerdos verbales con algunos asociados". (Prado, 1989, Pág. 133)

\subsection{La audiencia de la radio}

Pocos están de acuerdo en la total fiabilidad de los datos ofrecidos por los estudios de audiencia en España. La mayoría de los expertos admite que los sistemas de medición y control de los medios de comunicación presentan carencias. En la radio estas carencias se hacen más patentes porque ya hay serias dificultades para cuantificar el número de emisoras existente. Saber cuáles son operativas y cuántas emiten en cadena parcial o continuamente es la segunda parte de la oscura tarea. $\mathrm{Si}$ a ello se añade que los datos sobre las inversiones publicitarias en el medio son diferentes según sea su procedencia -anunciantes o cuentas de resultados de las propias cadenas-, no hay más remedio que aceptar que la radio se mueve en un terreno donde las cifras deben ser tomadas con cierta cautela.

Hecho este inciso, conviene reseñar los dos estudios que habitualmente se tenían en cuenta a principios de los noventa para el análisis y cuantificación de la audiencia de radio: el Estudio General de Medios y el estudio ICP/Radio, después. Sin embargo, a mediados de la década de los noventa, ICP/Radio deja de realizar el estudio.

Pero, ¿qué diferencia había entre el Estudio General de Medios y el estudio ICP/Radio?

El Estudio general de Medios (EGM) se realiza en tres oleadas de entrevistas. El planteamiento de este estudio de carácter multimedia, elaborado por la Asociación para la Investigación de los Medios de Comunicación (AIMC), es el recuerdo de la 
víspera. Las oleadas responden a tres encuestas anuales. En cada período se realizan unas 14.600 entrevistas personales, en el domicilio de los preguntados. A los encuestados se les pregunta sobre su comportamiento del día anterior en relación con los distintos medios de comunicación.

El estudio ICP/Radio era un estudio de audiencia específico del medio radio y las encuestas se hacían por teléfono. También se preguntaba por el comportamiento del día anterior. Según la empresa encargada del estudio, diariamente se hacían 100 entrevistas entre las seis de la tarde y las diez de la noche. Se trataba de un estudio continuado, y la información obtenida se iba incorporando al programa de clasificación de las emisoras -elaborado en colaboración con las propias empresas radiofónicas- que contaba, además, con el asesoramiento de Luis Vives Radio ${ }^{8}$.

Dicho esto conviene resaltar que desde 1982, la audiencia de la radio española había experimentado un continuo descenso. Las estimaciones sobre la pérdida de oyentes desde 1982 hasta 1988 se cifraba en casi nueve puntos porcentuales, lo que equivalía a que uno de cada siete oyentes había dejado de serlo. De acuerdo con el análisis de Eduardo García Matilla ${ }^{9}$ sobre la audiencia de radio durante 1988, las pérdidas de oyentes de la Onda Media en el período 1982-1988 podía calificarse de espectacular, puesto que la caída alcanzaba un $20 \%$ y situaba la audiencia, en 1988 , en el 21,45 de la población. Como altamente preocupante describía García Matilla el envejecimiento de la audiencia que seguía las emisiones de Onda Media, sobre todo porque al medio no se incorporaban jóvenes oyentes.

Del EGM de 1988 se desprendía que la audiencia de la FM se estancaba, hecho que supuso un hito en la década de los ochenta: por primera vez esta audiencia detenía su paulatino crecimiento. La cota alcanzada era similar a la de 1987 , el $34,6 \%$ de la población mayor de 14 años.

\footnotetext{
${ }^{8}$ VV.AA. "La radio en estudio" en Anuncios, num. 25. Marzo. 1992. (págs. 11 y 13).

${ }^{9}$ GARCÍA MATILLA, Eduardo y MADINAVEITTA, Eduardo. Recontr 1989. RNE. Madrid, 1989. (pág 140)
} 
En 1988, la audiencia de la radio bajó en las horas de mañana, el período de máxima escucha. Se perdían más de 700000 oyentes entre las 10:00 y las 13:00 horas. García Matilla, que atribuía este descenso a la puesta en marcha de la televisión matinal, advertía de la aparente contradicción que suponía que la inversión publicitaria en el medio siguiera incrementándose mientras se producía la paulatina pérdida de oyentes.

En la oleada final del EGM de 1989 se vuelve a observar el trasvase de oyentes de la OM a la FM. El promedio de audiencia por día semanal se situaba en el 53,3\%; es decir, en 16018000 oyentes, de los que el 20,5\% -6172000- se decantaba por la Onda Media y el resto por la FM.

Por lo que se refiere al litigio de las grandes cadenas por la audiencia, Mariano Cebrián Herreros ${ }^{10}$ destaca los altibajos de las emisoras, según las diferentes oleadas del EGM. Dentro de la OM, el primer lugar se intercambiaban la SER y RNE -entre 2000000 y 2500000 oyentes-, seguidos por la COPE, con menos de dos millones de oyentes. En la FM, el número se situaba en los 10800000.

Si el contraste se efectuaba según el modelo de programación, se observaba que en la programación de contenidos heterogéneos, para todos los públicos, se daban los siguientes datos: SER: 2823000; Radio 1 de RNE: 2664000: Antena 3: 2100000; COPE: 1816000; mientras que en la programación de radio fórmula el reparto era como sigue: SER: 3727000; COPE: 859000; Radio 80: 488000 y Radio Minuto: 336000 oyentes.

Según el informe FUNDESCO correspondiente a 1991, las emisoras del Grupo PRISA reunían el $43,69 \%$ de la audiencia semanal, seguidas de RNE, con el 19,12\%. El conjunto de la radiodifusión privada alcanzaba un volumen de audiencia del 80,88\%, mientras que la radio estatal se situaba en el 19,12\%. En la programación convencional, las emisoras estatales -Radio1 y Radio5- ocupaban el primer puesto

\footnotetext{
${ }^{10}$ CEBRIAN HERREROS, Mariano. "Una reconversión pendiente". En VV.AA. Comunicación Social: 1990. Tendencias. Fundesco. Madird. (pág. 160)
} 
con 2511000 oyentes, seguidas por la Cadena SER, con 2447000, y Antena 3, con 2095000 oyentes. A pesar de que la sintonía del medio radiofónico disminuyó un $4,4 \%$ en 1990 , las cadenas, en términos generales, sufrieron menos el descenso, que se cifró en 2,2 puntos porcentuales.

\subsection{La programación radiofónica}

El continuo descenso de la audiencia no fue una razón de peso para los programadores de los últimos años de la década de los ochenta, que fueron fieles a un reducido tipo de programas. Los informativos "despertadores", los programas "magazine" de mañana y tarde, los deportivos y los informativos de noche fueron el modelo de programación de la radio española de los años que aquí se analizan. Sin olvidar, por supuesto, la contratación de estrellas o grandes comunicadores con audiencia propia, tendencia que sigue imperando en la radio de nuestros días.

Los teóricos del medio García Matilla, Madinaveitia y Torre cervigón criticaron la escasa capacidad de riesgo de las empresas radiofónicas ${ }^{11}$. Pese al descenso ininterrumpido de audiencia, comprobado desde 1982, las empresas radiofónicas mantenían similares esquemas de programación. Estos esquemas se basaban en las estrellas, en Iso programas magazine en las horas punta $y$, salvo contadas excepciones, en una ausencia notable de capacidad de riesgo.

En estos años, la alternativa a la programación de la radio convencional era la FM, que continuó con la programación de radio fórmula, también llamada radio tocadiscos.

\footnotetext{
${ }^{11}$ PRADO, Emilio: "La radio española ante el desafío del futuro" en VV.AA. Comunicación social: 1989. Tendencias. Fundesco. Madrid. (pág. 132)
} 
La batalla de las diferentes emisoras por la audiencia se sustentaba en una competencia en la que no ofrecía diferenciación: se daban los mismos contenidos a las mismas horas. Entre las novedades, García Matilla ${ }^{12}$ hacia referencia a la Cadena SER, que en su programación de 1988 optó por el cambio en algunos segmentos horarios de su "target" de audiencia. La empresa líder buscaba, en las tardes y en los fines de semana por la mañana, interesar al público más joven y con un nivel cultural superior al habitual en esta cadena.

El refuerzo de los programas informativos fue otro de los objetivos que se marcaron las cadenas en la temporada de 1989. La SER, Antena 3, La COPE y RNE decidieron profundizar en la información, incluyendo en sus programas el reportaje en directo. También es ésta una tendencia arrastrada hasta las programaciones actuales, con una clara proyección hacia los formatos "All News" o "Todo Noticias", destino final de Radio 5, resultante del proceso de fusión.

En cuanto a programaciones especializadas, había algunas iniciativas como las de Radio Salud, en Barcelona; la de Radio 2, de RNE, de música clásica, o la de Radiolé más tarde, de música ligera española, en Madrid. La mayoría de los expertos de la radio auguraban una tendencia futura hacia las programaciones especializadas, tal y como ocurre en Estados Unidos y en algunos países europeos. Otros analistas argumentaban que la especialización supone menos oyentes $y$, por tanto, menos ingresos publicitarios. Frente a ello, Mariano Cebrián Herreros ${ }^{13}$ matizaba que

"...no se trata de quitar publicidad a otras emisoras, sino de buscar otra que no emerge porque no encuentra su público específico...Aquí se ha preferido mantener la oferta con los mismos contenidos que los demás y luchar todos por la misma tarta publicitaria". (Cebrián Herreros, 1992, Pág. 220)

\footnotetext{
${ }^{12}$ GARCÍA MATILLA, Eduardo y MADINAVEITTA, Eduardo. Recontr 1989. RNE. Madrid, 1989 (pág 144)

${ }^{13}$ CEBRIÁN HERREROS, Mariano. "radio pública/radio privada", en VV.AA. Comunicación social: 1992. Tendencias. Madrid. pág. 220.
} 
Según Mariano Cebrián, algo similar había ocurrido en la radio pública,

"que en lugar de esforzarse por atender a otras audiencias y ofrecer contenidos, prefirió, entonces, concurrir con las mismas estrategias que las radios comerciales, con grave detrimento de sus funciones de servicio a todos los públicos, mayoritarios y minoritarios" (Cebrián Herreros, 1992, Pág. 220)

En general, a los programadores de los últimos años ochenta se les reprochaba no haber sabido adaptarse a las demandas de la sociedad española. Varios expertos coinciden en citar el análisis que hacen García Matilla, Madinaveitia y Torre Cervigón ${ }^{14}$ sobre la ruptura de la radio y sus oyentes.

"la radio española, que en los años de transición fue uno de los motores fundamentales del cambio social, no ha sabido adaptarse a los gustos de la audiencia que ella misma contribuyó a transformar. La insistencia de las emisoras de radio convencional en dar "mas de lo mismo a las mismas horas" ha agotado las fórmulas que dieron juego en el pasado y ha propiciado el éxito como alternativa de las baratísimas radios-tocadiscos". (García Matilla y Madinaveitia 1989, Pág. 67)

\section{El entorno social e informativo para la fusión RCE/RNE}

Los últimos años de la década de los ochenta, cuando se produce la fusión entre Radio Nacional de España y Radiocadena Española, apuntaban hacia el protagonismo

\footnotetext{
${ }^{14}$ GARCÍA MATTILLA, Eduardo, MADINAVEITIA, Eduardo y TORRE CERVIGÓN, José Ma "Señales de alarma en la radio española. El "crack" del 92" en Revista Mensaje y Medios, no 3 Nueva Época. Ente público RTVE (pág. 7).
} 
de la información en la sociedad española pero, también, hacia un país con la crisis económica, el paro y la marginación bajo sus pies.

Esa importancia de la información y los aspectos económicos, justificaron, entonces, el nacimiento de una radio pública mucho más fuerte y con recursos más aprovechados, quizá como anticipo de la crisis financiera y social reflejada en esos índices de paro y marginación.

Repasemos cuál era el entorno social e informativo de la época.

\subsection{Aspectos sociales}

"La España del último decenio del siglo es la España de los 40 millones de almas" (De Miguel, 1992-93, Pág. 37), según se señala en el informe sociológico, dirigido por Amando de Miguel $^{15}$, La sociedad española 1992-93. La estimación del Instituto Nacional de Estadística (INE) de 1987 calculaba 39,4 millones de habitantes para 1990, cifra equivalente a la que certifica el censo de 1991.

La tasa bruta de natalidad -número de nacidos por mil habitantes- pasaba del $22 \%$ en 1960 al $11 \%$ en 1986, descenso muy similar al de los países del entorno cultural español (Francia, Italia, Irlanda, Grecia y Portugal). El dato es algo inferior al de Portugal y algo más elevado que el de Italia y Gracia. Mientras, la tasa bruta de mortalidad -fallecidos por mil habitantes- era la más baja de los seis países. Además, se detectaba una fuerte reducción del crecimiento vegetativo: nacidos menos fallecidos por mil habitantes. España se aproximaba al crecimiento cero de Italia al alcanzar un 2 por mil de crecimiento vegetativo anual en 1989.

\footnotetext{
${ }^{15}$ DE MIGUEL, Amando. "La sociedad española 1992-93". Informe sociológico de la Universidad Complutense. Alianza editorial. Madrid. (pág. 37).
} 


\subsection{El mundo laboral}

A la luz de las encuestas, el paro era una forma de marginación y una de las cuestiones que más preocupaba a los españoles. En la prensa abundaban los titulares relativos a cuestiones de empleo. Por ejemplo, se decía que a finales de 1989 el número de desempleados inscritos en el INEM ascendía a 2426572 (El País, 12 de enero de 1990) y que al final de 1990 la Encuesta de Población Activa (EPA) señalaba 2424320, el 16,11\% de la población activa /El País, 22 de febrero de 1991). El paro se interpretaba como una manera de estar al margen de la economía, y la lectura, independientemente de las cifras, era que afectaba a un gran número de personas. Además, en el desempleo se observaba una segunda forma de marginación: el trabajo sin contrato fijo. Los asalariados de la EPA de finales de 1990 aumentaron en relación a 1989, pero los contratos fijos habían disminuido en 64000 (El País, 22 de febrero de 1991).

El trabajo temporal era otra manera de estar al margen de la economía. Tres millones de personas se encontraban en esta situación (El País, 21 de noviembre de 1991). También la economía sumergida: entre 3 y 4 millones de españoles trabajaban en ella (El Independiente, 9 de marzo de 1990). La revista Cáritas (núm. 313 , 1991) calculaba que un $35 \%$ de la población activa contaba con empleo precario o inestable, y según el periódico El Sol (14 de octubre de 1991), un tercio de los parados carecía de preparación para encontrar empleo. Según el sociólogo Amando de Miguel "la marginación no equivalía a ser una fracción muy reducida de la población ${ }^{\prime 16}$. (De Miguel, 1992-93, Pág. 472)

\footnotetext{
${ }^{16}$ DE MIGUEL, Amando. "La sociedad española 1992-93". Informe sociológico de la Universidad Complutense. Alianza editorial. Madrid. (Pág. 472)
} 


\subsection{Niveles de instrucción y marginación}

Aparte de la marginación procedente de la situación en el mercado de trabajo, otra forma de estar al margen derivaba de la cultura. En ese sentido se debía tener en cuenta el analfabetismo. En España había casi dos millones de analfabetos absolutos, según Diario 16 (6 de junio de 1990). El periódico el Sol señalaba que eran más de un millón (28 de mayo de 1990), El Independiente (8 de noviembre de 1990), que había 12 millones de analfabetos totales o funcionales, y que la mitad de los analfabetos se encontraban en Andalucía, Valencia y Castilla-La Mancha.

\subsection{Aspectos informativos}

En los meses de gestación y ejecución de la fusión entre radio Nacional de España y radiocadena Española, tuvo lugar una interesante actividad informativa.

\subsubsection{Internacional}

La política internacional estaba marcada por los coletazos finales de la "era Reagan" y por la emergencia de un nuevo líder en la Unión Soviética -Mijail Gorbachov- capaz de acabar con el paradigma del Este. Francisco G. Basterra ${ }^{17}$ calificó a Gorbachov como responsable de salvar a Ronald Reagan del peor desastre de política exterior de su presidencia en 1987: el Irangate.

\footnotetext{
${ }^{17}$ G. BASTERRA, Francisco. "El final de la era Reagan" en Anuario El País 1988. Ediciones El País. (pág. 20).
} 
En 1988 se consiguieron importantes avances en el terreno de la paz en zonas conflictivas como África, Asia y oriente Próximo. Javier Pérez de Cuéllar ${ }^{18}$ subrayó la capacidad de las Naciones Unidas demostrada en la pacificación internacional.

"La concesión del Premio Novel de la paz de 1988 a las fuerzas de mantenimiento de la paz de las naciones Unidas constituyó un bien merecido tributo a su función permanente de protección multilateral de la paz" (Pérez De Cuéllar, 1989, Pág. 12), declaró Pérez de Cuéllar.

Del balance del año 1988, Pérez de Cuéllar destacaba las reuniones entre Gorvachov y el presidente Reagan orientadas a nuevas relaciones de trabajo entre ambos países. Apuntaba el ex secretario general de la ONU la eficacia de los avances conseguidos en la eliminación de conflictos violentos.

La economía mundial tuvo en 1988 un crecimiento superior al estimado a finales de 1987, cuando todavía estaba presente el efecto psicológico del "crash" bursátil de Nueva york acaecido en octubre. Según los cálculos de la Organización para la Cooperación y el desarrollo Económico (OCDE) y del fondo Monetario Internacional (FMI), el crecimiento económico superó en un 4\% al de 1987.

Las perspectivas económicas para 1989 mantenían la expansión registrada un año antes, aunque con ritmos de crecimiento más moderados. En este sentido, las previsiones indicaban un crecimiento del 3,5\% (frente al $4 \%$ en 1988), un aumento de medio punto en la inflación (el $3,5 \%$ en 1988) y el mantenimiento del desempleo en niveles similares a los 1988 (el 7,25\% en el área de la OCDE).

\footnotetext{
${ }^{18}$ PÉREZ DE CUELLAR, Javier "1988, El año de la paz". En Anuario El País 1989. Ediciones El País. (pág. 12).
} 


\subsubsection{Nacional}

En los meses de final de la década de los ochenta surgieron movimientos de protesta en el sector docente.

Los sindicatos de profesores de la enseñanza pública no universitaria anunciaban el 29 de febrero de 1988 la convocatoria de siete días de paros intermitentes en demanda de la homologación de sus retribuciones complementarias con los restantes funcionarios de la Administración, entre otras peticiones.

Al final, la convocatoria se convirtió en 20 días de paros que interrumpieron el desarrollo normal de los cuatro últimos meses del curso 1987-88. El conflicto propició la caída del ministro de Educación, José María Maravall, y su relevo por Javier Solana, hasta entonces responsable de la cartera de cultura. Los profesores, según el relato de Esteban S. Barcia ${ }^{19}$, "acusaron al equipo de José María Maravall de prepotencia, el gran pecado del Gobierno socialista y origen principal, según tantas interpretaciones, de la ya histórica huelga general del 14 de diciembre de 1988' (S. Barcia, 1989, Pág. 133).

Los sindicatos y el ministro Javier Solana suscribieron el 19 de noviembre de 1988 un acuerdo por el que el sistema retributivo del personal docente se integraba en el sistema retributivo general de los funcionarios públicos, recogiendo las especificidades de la función docente, y donde las retribuciones de los docentes estaban homologadas con las que percibían los funcionarios de igual categoría.

El año 1988 contemplaba, también, cómo Jordi Pujol conseguía revalidar la tercera victoria del centro-derecha nacionalista en las elecciones autonómicas catalanas el 29 de mayo de 1988. La Coalición Convergència i Unió (CiU) conseguía el 46,01\% de los

\footnotetext{
${ }^{19}$ S. BARCIA, Esteban "La huelga de la enseñanza pública" en Anuario El País 1989. Ediciones El País. (pág. 133).
} 
votos y 69 diputados. Los socialistas catalanes, con el $29,27 \%$ de los votos y 42 escaños, convalidaron su condición de primera fuerza política de la oposición.

El éxito de Pujol no colmó las expectativas de CiU, según constata Enric Company ${ }^{20}$, ya que "perseguía superar el 50\% de los votos, ampliar su grupo parlamentario hasta 75 diputados y alcanzar 1400000 sufragios" (Company, 1989, Pág 102).

También los movimientos internos registrados en los partidos políticos españoles durante 1988 fueron decisivos a la hora de valorar las actuales formaciones políticas.

En el partido en el gobierno, el $31^{\circ}$ Congreso del PSOE se celebró 10 meses antes de la huelga general del 14 de diciembre. Las discrepancias en la "familia socialista" se hicieron patentes en la clausura del congreso durante los discursos del secretario general del PSOE, Felipe Gonzáles, y el del sindicato UGT, Nicolás Redondo. Joaquín Prieto $^{21}$ recuerda el llamamiento de Felipe González a la concertación social entre empresarios, sindicatos y Gobierno, mientras que el líder ugetista advertía a sus delegados: "Si todo lo que los trabajadores esperamos del Gobierno socialista tenemos que pactarlo con los empresarios, es mejor tener las manos libres" (Prieto, 1989, Pág 69).

Además del enfrentamiento entre Redondo y González, el congreso socialista dio a luz propuestas como el Plan de Empleo Juvenil, al que se opusieron las centrales sindicales, y la cuota del $25 \%$ para la participación de las mujeres en los órganos de dirección del partido.

Un mes después se celebró el $12^{\circ}$ Congreso del Partido Comunista de España(PCE). Julio Anguita era el nuevo líder del PCE en sustitución de Gerardo Iglesias.

También, por esos días, el Presidente González inauguraba una nueva etapa de Gobierno. El cuarto Gobierno socialista, constituido el 7 de julio de 1988, cuenta con seis nuevos ministros. Al nuevo gabinete se incorporan dos mujeres: Matilde

\footnotetext{
${ }^{20}$ COMPANY, Enric. "La tercera victoria de Jordi Pujol" en Anuario El País 1989. Ediciones El País. (pág. 102)

${ }^{21}$ PRIETO, Joaquín. "Cambios en la política española" en Anuario El País 1988. Ediciones El País. (pág. 59).
} 
Fernández, como titular del Ministro de Asuntos Sociales -de nueva creación-, y Rosa Conde, la ministra Portavoz del Gobierno, cartera que se desgaja de la de Cultura. En el ministerio de Justicia, Enrique Múgica Herzog sustituyó a Fernando Ledesma. La cartera de Interior fue ocupada por José Luis Corcuera en lugar de José Barrionuevo, que pasó a encargarse de la de Transportes, Turismo y Comunicaciones, conducida por Abel Caballero en el tercer Gobierno socialista.

Felipe González nombró responsable del Ministerio de Educación y Ciencia a Javier Solana, anterior titular de la cartera de Cultura y Portavoz del Gobierno, que sucedió a José María Maravall. El nuevo ministro de Cultura es Jorge Semprúm.

Y, de igual modo, la Constitución española cumplía 10 años el 6 de diciembre de 1988. La constitución más larga gestación de nuestra historia. El catedrático de Ciencias Políticas Ignacio Sotelo ${ }^{22}$ destacaba que cualquier juicio que se haga respecto a la Constitución de 1978

"ha de tener en cuenta la historia constitucional que la precede, las circunstancias económicas, social y políticas en las que se elabora, así como la coyuntura constitucional y políticas en las que se elabora, así como la coyuntura constitucional y política de la Europa de nuestro entorno" (Sotelo, 1988, Pág. 59).

Señalaba el catedrático que el paso del régimen franquista al democráticoconstitucional sin solución de continuidad se produce "sin el menos deslizamiento del poder, pese a la coincidencia de la crisis económica con la política"23 (Sotelo, 1988, Pág. 60).

Del prestigio ganado por la Constitución fuera de nuestras fronteras se valoraba el consenso alcanzado, a pesar de que se negociaba desde posiciones muy distintas.

\footnotetext{
${ }^{22}$ SOTELO, Ignacio. "En los 10 años de la Constitución" en Anuario El País 1988. Ediciones El País (pág. 59).

${ }^{23}$ SOTELO, Ignacio. "En los 10 años de la Constitución" en Anuario El País 1988. Ediciones El País (Pág. 60).
} 
Por último, los acontecimientos deportivos internacionales, con participación española: la Eurocopa y los Juegos de Seúl. Por cierto, con un protagonismo poco brillante de los representantes españoles-

La Eurocopa 88 de fútbol, que comenzó el 10 de junio en Alemania, prometía ser uno de los campeonatos de más interés. La calidad de los ocho equipos que la disputan, tres de ellos campeones del mundo -Alemania, Inglaterra e Italia-, avalaba la expectativa. El resto del grupo lo componían Holanda, España, URSS, Irlanda y Dinamarca.

Los Juegos Olímpicos de Seúl se celebraron en la capital surcoreana del 10 al 28 de septiembre de 1988. A la cita olímpica acudían 9650 deportistas procedentes de 160 países. Cuba fue la única baja importante de una olimpiada que partía con la esperanza de universalidad que no habían logrado las anteriores reuniones desde Munich 1972. En Montreal 1976, re retiraron los países africanos; en Moscú 1980, faltó la mayoría del bloque occidental, y en Los Ángeles 1984, los ausentes fueron los socialistas. Juan José Fernández ${ }^{24}$ recuerda que 1988 pudo pasar a la historia como el año de la reunificación de las dos Coreas.

"Pero el Norte puso demasiados inconvenientes. Quiso una coorganización a la que no tenía derecho ya inicialmente y ante las ofertas del Comité Olímpico Internacional y de la propia Corea del Sur, dio siempre la entera sensación de que pedía imposibles para que no hubiera acuerdo" (Fernández, 1989, Pág. 324).

El atleta canadiense Ben Johnson -el atleta más rápido del mundo- fue la estrella fugaz de la Olimpíada de Seúl. A las 72 horas de vencer en los 100 metros lisos dio positivo en el control antidoping. 
Este era, en general, la perspectiva que se divisaba en el ámbito social e informativo durante el proceso de fusión entre las dos emisoras pública: Radio Nacional de España y Radiocadena Española.

\section{Bibliografía}

CEBRIAN HERREROS, Mariano. "Una reconversión pendiente" en VV.AA. "Comunicación Social: 1990". Tendencias. FUNDESCO. Madrid.

CEBRIAN HERREROS, Mariano. "Radio pública/radio privada" en VV.AA. "Comunicación Social: 1992". Tendencias. FUNDESCO. Madrid.

COMPANY, Enric. "La tercera victoria de Jordi Pujol" en VV.AA. "Anuario El País 1989". Ediciones El País. Madrid.

DE MIGUEL, Amando. "La sociedad española 1992-93". Informe sociológico de la Universidad Complutense. Alianza editorial. Madrid.

DÍAZ-NOSTY, Bernardo. "La radio en España. Entre la crisis de identidad y los grandes beneficios" en VV.AA. "Comunicación Social: 1989". Tendencias. FUNDESCO. Madrid.

DÍAZ NOSTY, Bernardo. "Radio: síntomas de recesión" en VV.AA. "Comunicación social: 1991". Tendencias. FUNDESCO. Madrid.

FERNÁNDEZ, Juan José. "¿Cómo creer ya en los grandes campeones?" en Anuario El País 1989. Ediciones El País. (pág. 324).

FRANQUET, Rosa. "Consolidación de las radios municipales" en VV.AA. "Comunicación Social: 1991". Tendencias. FUNDESCO. Madrid

GARCÍA BASTERRA, Francisco. "El final de la era Reagan" en VV.AA. "Anuario El País 1988". Ediciones El País 
GARCIA MATILLA, Eduardo. "Un año de transición para la radio española" en VV.AA. "Comunicación social: 1989". Tendencias FUNDESCO. Madrid.

GARCÍA MATILLA, Eduardo y MADINAVEITTA, Eduardo. Recontr 1989. RNE. Madrid, 1989

PÉREZ DE CUELLAR, Javier. "1988, El año de la paz" en VV.AA. "Anuario El País 1989". Ediciones El País.

PRADO, Emilio. "La radio española ante el desafío del futuro" en VV.AA. "Comunicación social: 1989". Tendencias. Fundesco. Madrid

PRIETO, Joaquín. "Cambios en la política española" en VV.AA. "Anuario El País 1988". Ediciones El País.

SÁNCHEZ BARCIA, Esteban. "La huelga de la enseñanza pública" en VV.AA. "Anuario El País 1989". Ediciones El País

SOTELO, Ignacio. "En los 10 años de la Constitución" en VV.AA. "Anuario El País 1988". Ediciones El País

VV.AA. "La radio en estudio" en Revista Anuncios, num. 25. Marzo. 1992 